\title{
Decoherence-assisted initialization of a resident hole spin polarization in a $p$-doped semiconductor quantum well
}

\author{
M. Kugler, ${ }^{1}$ K. Korzekwa, ${ }^{2}$ P. Machnikowski, ${ }^{2, *}$ C. Gradl, ${ }^{1}$ S. Furthmeier, ${ }^{1}$ M. Griesbeck, ${ }^{1}$ M. Hirmer, ${ }^{1}$ D. Schuh, ${ }^{1}$ \\ W. Wegscheider, ${ }^{3}$ T. Kuhn, ${ }^{4}$ C. Schüller, ${ }^{1}$ and T. Korn ${ }^{1, \dagger}$ \\ ${ }^{1}$ Institut für Experimentelle und Angewandte Physik, Universität Regensburg, D-93040 Regensburg, Germany \\ ${ }^{2}$ Institute of Physics, Wroctaw University of Technology, PL-50-370 Wroctaw, Poland \\ ${ }^{3}$ Solid State Physics Laboratory, ETH Zurich, CH-8093 Zurich, Switzerland \\ ${ }^{4}$ Institut für Festkörpertheorie, Westfälische Wilhelms-Universität, D-48149 Münster, Germany \\ (Received 18 May 2011; revised manuscript received 1 July 2011; published 30 August 2011)
}

\begin{abstract}
We investigate spin dynamics of resident holes in a $p$-modulation-doped $\mathrm{GaAs} / \mathrm{Al}_{0.3} \mathrm{Ga}_{0.7} \mathrm{As}$ single quantum well. Time-resolved Faraday and Kerr rotation, as well as resonant spin amplification, are utilized in our study. We observe that nonresonant or high-power optical pumping leads to a resident hole spin polarization with opposite sign with respect to the optically oriented carriers, while low-power resonant optical pumping only leads to a resident hole spin polarization if a sufficient in-plane magnetic field is applied. The competition between two different processes of spin orientation strongly modifies the shape of resonant spin amplification traces. Calculations of the spin dynamics in the electron-hole system are in good agreement with the experimental Kerr rotation and resonant spin amplification traces and allow us to determine the hole spin polarization within the sample after optical orientation, as well as to extract quantitative information about spin dephasing processes at various stages of the evolution.
\end{abstract}

DOI: 10.1103/PhysRevB.84.085327

PACS number(s): 78.67.De, 78.55.Cr, 78.47.D-

\section{INTRODUCTION}

The promising field of semiconductor spintronics ${ }^{1}$ has stimulated a large number of studies of spin dynamics and spin dephasing mechanisms in a vast variety of semiconductors and their heterostructures in recent years. Especially the dynamics of conduction-band electrons in compound semiconductors without inversion center, for example, GaAs, have been studied by many groups (see, e.g., Ref. 2 for a recent review). By contrast, hole spin dynamics in these systems have been investigated with less intensity. This is, in part, due to the subpicosecond hole spin dephasing time (SDT) in bulk $\mathrm{GaAs},{ }^{3,4}$ which arises from the strong spin-orbit coupling within the $p$-like valence bands. In $p$-doped quantum wells (QWs), an increase of the hole SDT to a few picoseconds has been observed experimentally by several groups ${ }^{5,6}$ and reproduced in microscopic calculations. ${ }^{7}$ Significantly longer hole SDTs have been observed for optically oriented holes in $n$-doped QWs ${ }^{8,9}$ and more recently in $p$-doped QW systems in which localization of holes occurs at low temperatures ${ }^{10-13}$ and also in quantum dots. ${ }^{14}$ For localized electrons in quantum dots, the contact hyperfine interaction leads to ensemble spin dephasing on the 10 -ns scale. ${ }^{15}$ However, due to their $p$-like wave functions, this dephasing process is suppressed for holes, and only the weaker dipole-dipole interaction has to be taken into account. ${ }^{16}$ Therefore, localized holes may be more suitable than electrons for the realization of future quantum computing schemes. Additionally, the large orientational anisotropy of the hole $g$ factor in GaAs-based heterostructure ${ }^{17}$ strongly influences hole spin dynamics in tilted magnetic fields ${ }^{18}$ and may allow for spin manipulation schemes based on electrical $g$-factor control. ${ }^{19,20}$

Here we present time-resolved studies of the combined electron and hole spin dynamics in a $p$-modulation-doped QW under different excitation conditions. We utilize time-resolved Kerr/Faraday rotation, ${ }^{21}$ as well as the related resonant spin amplification technique, ${ }^{22}$ and time-resolved photoluminescence. We identify two processes in which spin polarization is transferred to the resident holes after optical orientation, quantitatively model the dynamics, and determine the contributions of these processes depending on excitation conditions. By fitting our theoretical model to the experimental results, we are able to find the degree of spin polarization after the optical excitation and to extract the hole SDTs, as well as the degree of coherence loss during excitation at various conditions of optical pumping. We determine transverse SDTs $T_{2}$ of almost $100 \mathrm{~ns}$ under weak resonant excitation. Additionally, we show that fast dephasing of the hole spin state during and just after high-power or blue-detuned pumping leads to polarization of the hole spins at zero magnetic field, while at finite fields this process competes with the polarization mechanism due to trion spin precession. ${ }^{10,20}$ As these two competing processes lead to opposite spin orientation our findings show that the spin orientation can be controlled by modifying the optical excitation conditions.

The paper is organized as follows. First, in Sec. II, we present the sample and the idea of the experiment. Next, in Sec. III, the theoretical model is introduced. Section IV contains the presentation and discussion of the experimental results and their theoretical modeling. Finally, Sec. V concludes the paper.

\section{SAMPLE STRUCTURE AND EXPERIMENTAL METHODS}

Our samples are single-side $p$-modulation-doped GaAs $/ \mathrm{Al}_{0.3} \mathrm{Ga}_{0.7} \mathrm{As}$ QWs (QW width $4 \mathrm{~nm}$ ), containing a two-dimensional hole system (2DHS) with a hole density $p=1.1 \times 10^{11} \mathrm{~cm}^{-2}$ and mobility $\mu=1.3 \times 10^{4} \mathrm{~cm}^{2} / \mathrm{Vs}$ (measured at $1.3 \mathrm{~K}$ ) from a single wafer grown by molecular beam epitaxy. Some samples from this wafer are thinned for 
measurements in transmission. For this, the samples are first glued onto a sapphire substrate with optically transparent glue, then the semiconductor substrate is removed by grinding and selective wet etching. The samples contain a short-period GaAs/AlGaAs superlattice, which serves as an etch stop, leaving only the MBE-grown layers. Earlier studies of spin dynamics performed on similar systems ${ }^{10,20}$ indicate that in structures of this kind the resident holes are weakly trapped, most likely on QW width fluctuations. This is confirmed by a rapid increase of hole spin dephasing above a certain threshold temperature, associated with thermal release of the carriers from these binding centers and the onset of spin-orbit-related dephasing characteristic of free carriers.

The resonant spin amplification (RSA) measurements are performed in an optical cryostat with ${ }^{3} \mathrm{He}$ insert, allowing us to lower the sample temperatures below $400 \mathrm{mK}$ and to apply magnetic fields of up to $11.5 \mathrm{~T}$. Here, the samples are cooled by cold ${ }^{3} \mathrm{He}$ gas. For some of these measurements, thinned samples are used and the experiment is performed in transmission (Faraday rotation) to limit the amount of absorbed laser power. The time-resolved Kerr rotation (TRKR) measurements are performed in a helium flow cryostat, in which the samples are mounted on the cold finger of the cryostat in vacuum. A pulsed Ti-sapphire laser system generating pulses with a length of $600 \mathrm{fs}$ and a spectral width of 3-4 meV is used for the optical measurements. The repetition rate of the laser system is $80 \mathrm{MHz}$, corresponding to a time delay of $12.5 \mathrm{~ns}$ between subsequent pulses. The laser pulses are split into a circularly polarized pump beam and a linearly polarized probe beam by a beam splitter. A mechanical delay line is used to create a variable time delay between pump and probe. Both beams are focused to a diameter of about $80 \mu \mathrm{m}$ on the sample using an achromat.

In the TRKR and RSA experiments, the circularly polarized pump beam is generating electron-hole pairs in the QW, with spins aligned parallel or antiparallel to the beam direction, that is, the QW normal, depending on the helicity of the light. In the TRKR measurements, the spin polarization created perpendicular to the sample plane by the pump beam, is probed by the time-delayed probe beam via the Kerr effect: The axis of linear polarization of the probe beam is rotated by a small angle, which is proportional to the out-of-plane component of the spin polarization. ${ }^{18,23}$ This small angle is detected using an optical bridge. A lock-in scheme is used to increase sensitivity.

The RSA technique is based on the interference of spin polarizations created in a sample by subsequent pump pulses. It requires that the SDT be comparable to the time delay between pump pulses. For certain magnetic fields applied in the sample plane, the optically oriented spin polarization precesses by an integer multiple of $2 \pi$ in the time window between subsequent pump pulses, so that constructive interference occurs. This leads to pronounced maxima in the Faraday or Kerr rotation angle measured for a fixed time delay as a function of the applied magnetic field. In our measurements, the time delay is chosen to probe the spin polarization remaining within the sample 100 ps before the arrival of a pump pulse.

Time-resolved photoluminescence (TRPL) measurements are performed using a Hamamatsu streak camera system synchronized to the pulsed Ti-sapphire laser system. For these measurements, the laser is detuned to create electron-hole pairs

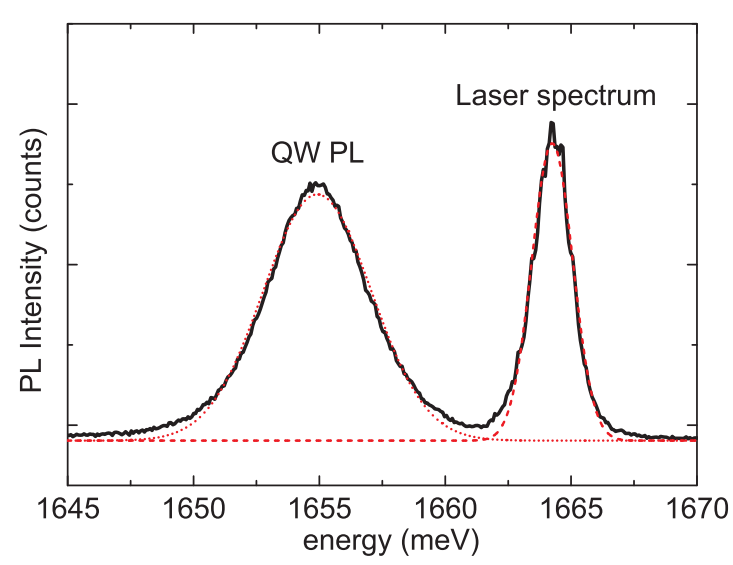

FIG. 1. (Color online) Photoluminescence (PL) trace of the sample measured at $1.2 \mathrm{~K}$ and spectrum of the Ti-sapphire laser system used in the time-resolved experiments. The broken lines show Gaussian fits to the QW PL (dotted line) and the laser spectrum (dashed line).

at an energy about $30 \mathrm{meV}$ above the photoluminescence (PL) energy of the heavy-hole exciton and trion lines. The PL from the sample is collected using an achromat and dispersed in a spectrometer before being detected by the streak camera.

For initial characterization of the samples, PL measurements using continuous-wave excitation with a 532-nm laser are performed. A grating spectrometer with a Peltier-cooled CCD detector is used to collect the PL. Figure 1 shows a typical PL trace measured at a sample temperature of $1.2 \mathrm{~K}$. The PL from the QW is a near-symmetrical single peak with a spectral width of about $5 \mathrm{meV}$. No fine structure of this peak, corresponding to, for example, neutral or positively charged excitons, is observed in PL measurements. In the same figure, a typical spectrum of the Ti-sapphire laser system can be seen at higher energy. In the following, by "resonant excitation" we mean the situation where the Ti-sapphire laser system is tuned to achieve maximum Kerr signal. Since our sample is doped, the states corresponding to the maximum of the PL emission are occupied by resident holes; therefore, the absorption at this energy is suppressed. Maximum Kerr signal is observed for an energetic position of the laser at the high-energy flank of the QW PL emission.

\section{THEORETICAL MODEL}

In order to interpret the experimental results we propose a minimal, generic model that is able to account for all the features of the spin dynamics observed in the experiment without specific assumptions on the detailed mechanism of spin decoherence. In accordance with the previous experimental findings, ${ }^{10,20}$ we assume that the optical response can be described in terms of independent hole-trion systems, trapped in QW fluctuations. The state of each such system is represented by the density matrix $\rho$, restricted to the four relevant states, $|\uparrow\rangle,|\downarrow\rangle,|T \uparrow\rangle,|T \downarrow\rangle$, representing the two hole states and the two trion states with different spin orientations (with respect to the system symmetry axis, normal to the sample plane). We neglect the influence of the weak probe pulse on the spin dynamics and calculate the spin polarization 
at the arrival of the probe, which is known ${ }^{18,23}$ to be translated by the probe into the Kerr or Faraday signal.

The experiment is modeled by a sequence of three steps as follows.

First, the pump pulse transforms the initial state $\rho_{0}$ into a new state $\rho_{1}$, described up to the second order in the pulse amplitude by

$$
\begin{aligned}
\rho_{1}= & -\frac{i}{\hbar} \int_{-\infty}^{\infty} d t\left[H_{1}(t), \rho_{0}\right] \\
& -\frac{1}{\hbar^{2}} \int_{-\infty}^{\infty} d t \int_{-\infty}^{\infty} d t^{\prime}\left[H_{1}(t),\left[H_{1}\left(t^{\prime}\right), \rho_{0}\right]\right],
\end{aligned}
$$

where we use the fact that the pulse is very short compared to the spin evolution time scales and assume, for simplicity, that the excitation is coherent. Here $H_{1}=(1 / 2) f(t)|\uparrow\rangle\langle T \uparrow|+$ H.c. is the carrier-laser coupling Hamiltonian with a pulse envelope $f(t)$ and $\sigma^{+}$circular polarization is assumed for the laser pulse.

Second, we allow for a fast partial decoherence of the hole spin which takes place on time scales much shorter than the subsequent spin dynamics and is therefore modeled as instantaneous. This leads to a system state $\rho_{2}$ with

$$
\left\langle\downarrow\left|\rho_{2}\right| \uparrow\right\rangle=\left\langle\downarrow\left|\rho_{1}\right| \uparrow\right\rangle e^{-u / 2-w}
$$

and

$$
\left\langle\alpha\left|\rho_{2}\right| \alpha\right\rangle=\frac{1}{2}\left\langle\uparrow\left|\rho_{1}\right| \uparrow\right\rangle\left(1 \pm e^{-u}\right)+\frac{1}{2}\left\langle\downarrow\left|\rho_{1}\right| \downarrow\right\rangle\left(1 \mp e^{-u}\right),
$$

where $\alpha=\uparrow, \downarrow$ and the upper (lower) sign is for $\alpha=\uparrow(\downarrow)$. The factors $e^{-u}$ and $e^{-w}$ describe the effects of occupation relaxation and additional pure dephasing in the reference frame associated with the system symmetry axis (coinciding with the axis of optical orientation), which is the relevant one here in view of the fast character of the process, as compared to the Larmor precession.

It should be noted that the combination of coherent excitation and instantaneous dephasing does not necessarily reflect the actual microscopic kinetics of the system. In particular, for off-resonant excitation, hole spin flips during relaxation to low-energy states are also possible. While this process is clearly beyond our four-level model, its essential effect is bringing the hole spin polarization toward equilibrium and dephasing of the hole spin coherence. Both these effects are included in our model in terms of the instantaneous relaxation and dephasing factors $e^{-u}$ and $e^{-w}$.

In the third stage, the system evolution (Larmor precession, recombination and spin decoherence) is modeled in terms of the Markovian master equation (in the Schrödinger picture with respect to the spin dynamics but in the rotating frame with respect to the interband transition energy)

$$
\dot{\rho}=-\frac{i}{\hbar}\left[H_{0}, \rho\right]+\mathcal{L}_{\mathrm{h}}[\rho]+\mathcal{L}_{\mathrm{t}}[\rho]+\mathcal{L}_{\mathrm{r}}[\rho],
$$

with the initial condition $\rho(0)=\rho_{2}$. Here

$$
H_{0}=-\frac{1}{2} \mu_{\mathrm{B}} \boldsymbol{B} \hat{g}_{\mathrm{h}} \boldsymbol{\sigma}_{\mathrm{h}}-\frac{1}{2} g_{\mathrm{t}} \mu_{\mathrm{B}} \boldsymbol{B} \cdot \boldsymbol{\sigma}_{\mathrm{t}}
$$

is the hole and trion spin Hamiltonian, where $\mu_{\mathrm{B}}$ is the Bohr magneton, $\hat{g}_{\mathrm{h}}$ is the hole Landé tensor, $g_{\mathrm{t}}$ is the Landé factor of the trion (i.e., essentially, of the electron), which we assume to be isotropic, and $\sigma_{\mathrm{h}}, \sigma_{\mathrm{t}}$ are the vectors of Pauli matrices corresponding to the hole and trion spin, respectively (the hole is treated as a pseudo-spin-1/2 system), in the basis of hole spin states $|\uparrow\rangle,|\downarrow\rangle$ and trion spin states $|T \uparrow\rangle,|T \downarrow\rangle$. This Hamiltonian accounts for the spin pression with the Larmor frequencies $\omega_{\mathrm{h}}=\mu_{\mathrm{B}}\left|\hat{g}_{\mathrm{h}} \boldsymbol{B}\right| / \hbar$ and $\omega_{\mathrm{t}}=\mu_{\mathrm{B}} g_{\mathrm{t}} B / \hbar$ for the hole and trion, respectively.

The hole dissipator $\mathcal{L}_{\mathrm{h}}$ is obtained within the standard weak-coupling approach ${ }^{24}$ from the hole spin-environment Hamiltonian $H_{\mathrm{he}}=\boldsymbol{\sigma} \cdot \boldsymbol{R}^{(\mathrm{h})}$, where $\boldsymbol{R}^{(\mathrm{h})}$ are certain environment operators. We derive the evolution equation for the hole spin in the Markov limit as in Ref. 18 but without the secular approximation which does not hold in the general case of possibly low or vanishing magnetic fields. As a result, we get the dissipator (in the Schrödinger picture) in the form

$$
\begin{aligned}
\mathcal{L}_{\mathrm{h}}[\rho]= & -\pi \sum_{l j}\left[R_{l j}^{(\mathrm{h})}\left(\omega_{j}\right)\left(\sigma_{l} \sigma_{j} \rho-\sigma_{j} \rho \sigma_{l}\right)\right. \\
& \left.+R_{l j}^{(\mathrm{h})}\left(-\omega_{l}\right)\left(\rho \sigma_{l} \sigma_{j}-\sigma_{j} \rho \sigma_{l}\right)\right],
\end{aligned}
$$

where $l, j= \pm, 0, \omega_{0}=0, \omega_{+}=-\omega_{-}=\omega_{\mathrm{h}}$, and $\sigma_{ \pm, 0}$ are Pauli matrices in the reference frame associated with the $x$ direction (the orientation of the field),

$$
\sigma_{0}=\sigma_{x}, \quad \sigma_{+}=\sigma_{-}^{\dagger}=\frac{-\sigma_{z}+i \sigma_{y}}{2} .
$$

The spectral densities for the hole reservoir are

$$
R_{l j}^{(\mathrm{h})}(\omega)=\frac{1}{2 \pi \hbar^{2}} \int d t e^{i \omega t}\left\langle R_{l}^{(\mathrm{h})}(t) R_{j}^{(\mathrm{h})}\right\rangle, \quad l, j= \pm, 0,
$$

where $R_{0}^{(\mathrm{h})}=R_{x}^{(\mathrm{h})}, R_{+}^{(\mathrm{h})}=R_{-}^{(\mathrm{h}) \dagger}=-R_{z}^{(\mathrm{h})}-i R_{y}^{(\mathrm{h})}$, and $R_{l}^{(\mathrm{h})}(t)$ denotes the operator in the interaction picture with respect to the environment Hamiltonian. Consistently with the assumed $C_{4 \mathrm{v}}$ symmetry of the system, we set $R_{\alpha \beta}^{(\mathrm{h})}(\omega)=0$ for $\alpha, \beta=$ $x, y, z, \alpha \neq \beta$, and $R_{y y}(\omega)=R_{x x}(\omega)$. The trion spin dissipator $\mathcal{L}_{\mathrm{t}}$ is obtained in the same way with a set of trion-related spectral densities $R_{\alpha \beta}^{(\mathrm{t})}(\omega)$. We assume that the reservoirs coupled to electron (trion) and hole spins are uncorrelated.

The last term in Eq. (2) is the standard spontaneous emission generator that accounts for the radiative recombination of the trion (see Ref. 18) with the rate $\gamma_{R}$.

Equation (2) can be rewritten in terms of the three components of the hole spin polarization:

$$
\begin{gathered}
X_{\mathrm{h}}=\langle\uparrow|\rho| \downarrow\rangle+\langle\downarrow|\rho| \uparrow\rangle, \quad Y_{\mathrm{h}}=i(\langle\uparrow|\rho| \downarrow\rangle-\langle\downarrow|\rho| \uparrow\rangle), \\
\Sigma_{\mathrm{h}}=\langle\uparrow|\rho| \uparrow\rangle-\langle\downarrow|\rho| \downarrow\rangle
\end{gathered}
$$

(and analogous for the trion). For the hole spin polarization, the equations of motion are

$$
\begin{gathered}
\dot{X}_{\mathrm{h}}=-\left(\kappa_{z}+\kappa_{x}\right) X_{\mathrm{h}}+\left(\kappa_{x}^{\prime}+\kappa_{z}^{\prime}\right) N_{\mathrm{h}}, \\
\dot{Y}_{\mathrm{h}}=\omega_{\mathrm{h}} \Sigma_{\mathrm{h}}-\left(\kappa_{x 0}+\kappa_{z}\right) Y_{\mathrm{h}}, \\
\dot{\Sigma}_{\mathrm{h}}=-\omega_{\mathrm{h}} Y_{\mathrm{h}}-\left(\kappa_{x}+\kappa_{x 0}\right) \Sigma_{\mathrm{h}}+\gamma_{\mathrm{R}} \Sigma_{\mathrm{t}},
\end{gathered}
$$

where $N_{\mathrm{h}}$ is the hole population and

$$
\begin{gathered}
\kappa_{\alpha}=2 \pi\left[R_{\alpha \alpha}^{(\mathrm{h})}\left(\omega_{\mathrm{h}}\right)+R_{\alpha \alpha}^{(\mathrm{h})}\left(-\omega_{\mathrm{h}}\right)\right], \quad \kappa_{\alpha 0}=4 \pi R_{\alpha \alpha}^{(\mathrm{h})}(0), \\
\kappa_{\alpha}^{\prime}=2 \pi\left[R_{\alpha \alpha}^{(\mathrm{h})}\left(\omega_{\mathrm{h}}\right)-R_{\alpha \alpha}^{(\mathrm{h})}\left(-\omega_{\mathrm{h}}\right)\right],
\end{gathered}
$$

for $\alpha=x, z$. In order to find an interpretation of the dephasing rates appearing in Eqs. (3a)-(3c) we note that at $\boldsymbol{B}=0$ one 
has $\omega_{\mathrm{h}}=0$; hence, $\kappa_{\alpha}=\kappa_{\alpha 0}$ and the decoherence time for the spin polarization along the structure axis is $T_{z}^{(0)}=1 /\left(2 \kappa_{x 0}\right)$, while the decoherence time for the in-plane components of the spin polarization is $T_{x y}^{(0)}=1 /\left(\kappa_{z}+\kappa_{x 0}\right)$. On the other hand, in sufficiently strong fields (for $\omega_{\mathrm{h}} \gg \kappa_{\alpha}, \kappa_{\alpha 0}$ ), the longitudinal (with respect to the field orientation) spin relaxation time is $T_{1}=1 /\left(\kappa_{z}+\kappa_{x}\right)$ and the transverse relaxation (dephasing) time is $T_{2}=2 /\left(\kappa_{z}+\kappa_{x}+2 \kappa_{x 0}\right)$.

The equations of motion for the trion spin polarization are

$$
\begin{gathered}
\dot{X}_{\mathrm{t}}=-\left(\mu_{z}+\mu_{x}\right) X_{\mathrm{t}}+\left(\mu_{x}^{\prime}+\mu_{z}^{\prime}\right) N_{\mathrm{t}}-\gamma_{\mathrm{R}} X_{\mathrm{t}}, \\
\dot{Y}_{\mathrm{t}}=\omega_{\mathrm{t}} \Sigma_{\mathrm{t}}-\left(\mu_{x 0}+\mu_{z}\right) Y_{\mathrm{t}}-\gamma_{\mathrm{R}} Y_{\mathrm{t}}, \\
\dot{\Sigma}_{\mathrm{t}}=-\omega_{\mathrm{t}} Y_{\mathrm{t}}-\left(\mu_{x}+\mu_{x 0}\right) \Sigma_{\mathrm{t}}-\gamma_{\mathrm{R}} \Sigma_{\mathrm{t}},
\end{gathered}
$$

where $\mu_{\alpha}, \mu_{\alpha}^{\prime}$, and $\mu_{\alpha 0}$ are the trion decoherence rates defined as in Eqs. (4a) and (4b), but with the trion-related spectral densities $R_{\alpha \alpha}^{(\mathrm{t})}(\omega)$ taken at the trion Larmor frequency $\omega_{\mathrm{t}}$, and $N_{\mathrm{t}}$ is the trion occupation.

The optical response, that is, the rotation of the polarization plane of the reflected or transmitted probe pulse, is proportional to 18,23

$$
\Delta \Sigma=\Sigma_{\mathrm{t}}-\Sigma_{\mathrm{h}} .
$$

As the time-resolved Kerr response is investigated for experimental conditions of relatively high spin dephasing and relaxation rates, one can assume that the evolution after each laser repetition is independent and starts from the thermal equilibrium state. After the pump pulse, the trion and hole spin polarizations are

$$
\Sigma_{\mathrm{t}}=-\Sigma_{\mathrm{h}}=\Sigma^{(0)} .
$$

As a consequence of the initial dephasing, the hole spin polarization is reduced to

$$
\Sigma_{\mathrm{h}}^{(0)}=-\Sigma^{(0)} e^{-u}
$$

(we assume no fast dephasing of the trion spin polarization). Then, by solving Eq. (2) one gets the Kerr signal at $\boldsymbol{B}=0$ in the form

$$
\Delta \Sigma^{(\mathrm{Kerr})}=a e^{-\gamma_{\mathrm{t}} t}-b e^{-\gamma_{\mathrm{h}} t},
$$

where $a=(1+\eta) \Sigma_{\mathrm{t}}^{(0)}, b=\Sigma_{\mathrm{h}}^{(0)}+\eta \Sigma_{\mathrm{t}}^{(0)}, \eta=\gamma_{\mathrm{R}} /\left[\gamma_{\mathrm{t}}-\gamma_{\mathrm{h}}\right]$. Here $\gamma_{\mathrm{h}}=\kappa_{x}+\kappa_{x 0}$ is the hole spin decoherence rate and $\gamma_{\mathrm{t}}=$ $\mu_{x}+\mu_{x 0}+\gamma_{\mathrm{R}}$ is the trion spin decoherence rate. Since we do not propose any specific microscopic mechanism for the spin decoherence the rates $\gamma_{\mathrm{t}}, \gamma_{\mathrm{h}}$, and $\gamma_{\mathrm{R}}$ are treated as independent parameters of the model.

For the RSA signal, the spin polarization surviving between subsequent laser repetitions is essential. In order to find the resonantly amplified spin polarization just before the pump pulse, we find the mapping of the hole spin-related variables $X_{\mathrm{h}}, Y_{\mathrm{h}}, \Sigma_{\mathrm{h}}$ corresponding to the three-step state transformation described above, assuming that trion occupations and interband coherences decay completely in the repetition interval. Moreover, the RSA measurements are performed under conditions of long SDT; hence, we assume that the hole spin dephasing rates are small compared to the trion recombination rate. The RSA signal is then found as the fixed point of this three-step transformation to the leading (second) order in the pulse area, that is, in the weak excitation limit. The resulting spin polarization just before the arrival of the pump pulse is proportional to

$$
\Delta \Sigma^{(\mathrm{RSA})} \sim f \frac{P}{Q},
$$

where

$$
\begin{gathered}
f=1-e^{-u}-\frac{\omega_{\mathrm{t}}^{2}}{\gamma_{\mathrm{R}}^{2}+\omega_{\mathrm{t}}^{2}}, \\
P=\left(i \tilde{\omega}+\kappa^{\prime}\right) e^{i \tilde{\omega} t_{\mathrm{r}} / 2}-i \tilde{\omega} e^{-u / 2-w-\kappa t_{\mathrm{r}} / 2}-(\tilde{\omega} \rightarrow-\tilde{\omega}), \\
Q=e^{-u} P+\left[\left(i \tilde{\omega}-\kappa^{\prime}\right) e^{-u / 2-w+i \tilde{\omega} t_{\mathrm{r}} / 2}-i \tilde{\omega} e^{\kappa t_{\mathrm{r}} / 2}\right. \\
-(\tilde{\omega} \rightarrow-\tilde{\omega})] .
\end{gathered}
$$

Here $t_{\mathrm{r}}$ is the laser repetition period, $\kappa=\kappa_{x}+\kappa_{z}+2 \kappa_{x 0}$, $\kappa^{\prime}=\kappa_{z}-\kappa_{x}, \tilde{\omega}=2 \sqrt{\omega_{\mathrm{h}}^{2}-\kappa^{\prime 2} / 4}$, and $(\tilde{\omega} \rightarrow-\tilde{\omega})$ represents additional terms, obtained from the preceding ones by changing the sign of $\tilde{\omega}$. It is found that the RSA response in the weak excitation limit does not directly depend on detuning. In order to simulate the response from an inhomogeneous ensemble of hole spins, the result obtained from Eqs. (8a)-(8d) was averaged according to a Gaussian distribution of hole $g$ factors with the standard deviation $\Delta g$.

\section{RESULTS AND DISCUSSION}

In this section we present the results of TRKR and RSA measurements and interpret them, based on the theoretical model presented in the previous section. We first discuss the Kerr measurements at zero magnetic field and then the RSA results.

\section{A. Kerr response at $B=0$}

First, we investigate the TRKR measurements at zero magnetic field. Here we performed two series of measurements: as a function of the pump frequency detuning and as a function of the pump pulse power.

In the frequency detuning series, performed at a fixed sample temperature of $15 \mathrm{~K}$, the excitation wavelength was tuned from near-resonant excitation to higher laser energy, which resulted in nonresonant excitation conditions. Figure 2(a) shows three TRKR traces for resonant excitation and two different values of laser energy detuning (symbols). While the trace for resonant excitation shows a near-monoexponential decay, the two traces measured using larger laser energies display a more complex behavior, with a very rapid initial decay of the signal and a zero crossing, followed by a slower decay of the negative signal. Additionally, we note that the Kerr signal amplitude decreases as the laser energy is detuned from the resonance, limiting the detuning range accessible in the measurements to about $7 \mathrm{meV}$. This is due both to a reduced absorption of the pump pulse and to the spectral dependence of the Kerr rotation of the degenerate probe pulse.

We interpret the traces as follows: Under resonant excitation conditions in the absence of a magnetic field, both the optically oriented electrons and the optically oriented holes retain their spin orientation during the photocarrier lifetime. Electrons recombine with holes that have a matching spin orientation according to the selection rules, thereby removing the optically created spin polarization from the sample during 

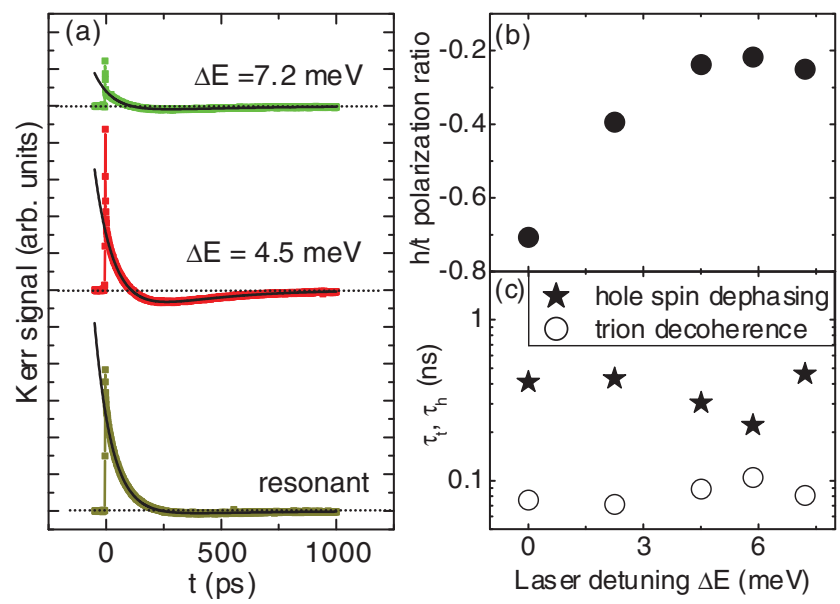

FIG. 2. (Color online) (a) TRKR traces measured at $15 \mathrm{~K}$ with different laser excitation energies (symbols). The solid lines represent fits to the data according to the theory (extended to $t<0$ for better visibility). (b) Ratio of hole and trion spin polarizations after rapid initial hole dephasing, $\Sigma_{\mathrm{h}}^{(0)} / \Sigma_{\mathrm{t}}^{(0)}=-e^{-u}$, extracted from the measurements as a function of laser detuning. (c) Hole SDT (solid stars) and trion spin decoherence time (open symbols) as a function of laser detuning.

recombination. Therefore, no hole spin polarization is transferred to the resident holes. By contrast, under nonresonant excitation conditions, a fraction of the hole spin polarization is rapidly lost. This can result from hole spin flips during energy relaxation of the optically created holes or from the thermalization of the spin orientation of the resident holes resulting from binding part of the oppositely oriented holes with the optically created excitons into trions. On the other hand, the electrons seem to retain their spin orientation. Upon recombination, these spin-polarized electrons remove holes with matching spin polarization from the partly depolarized hole system, leaving an excess of holes oriented opposite to the optically created hole spin orientation (we will refer to this opposite orientation as negative). We note that both the spin-polarized electrons and holes created by interband absorption of circularly polarized light will lead to the same Kerr rotation of a test beam, so that a priori, the observed Kerr rotation does not allow us to identify the type of spin-polarized carriers directly. The origin of the Kerr signal can be determined by applying a magnetic field perpendicular to the spin polarization and observing the spin precession, using the different $g$ factors of electrons and holes. Naturally, in the case of a doped sample like our 2DHS, investigating the Kerr rotation after photocarrier recombination, so that only the resident carriers remain, also gives unambiguous results.

The experimental traces are well-reproduced by Eq. (7) [the fits are shown as solid lines in Fig. 2(a)] in the whole time range except for the first few picoseconds after excitation, in which the rapid initial dephasing of the holes occurs, which is not modeled in a time-resolved manner in the theory. From the least-squares fit parameters, we are able to extract the ratio of the hole and electron (trion) spin polarizations, $\Sigma_{\mathrm{h}}^{(0)} / \Sigma_{\mathrm{t}}^{(0)}=-e^{-u}$ [see Eq. (6)] after the initial dephasing, before photocarrier recombination occurs. Figure 2(b) shows the calculated results as a function of the laser energy detuning from resonance. We see that, for resonant excitation, the ratio is close to -1 , indicating a hole spin polarization almost equal to and oriented opposite to the electron spin polarization. As the laser energy is increased, this ratio is reduced significantly but does not reach zero, indicating that some part of the optically oriented holes retain their spin orientation during energy relaxation. For all values of the detuning, we also extract the trion (electron) spin decoherence time $\tau_{\mathrm{t}}=1 / \gamma_{\mathrm{t}}$, and the hole SDT, $\tau_{\mathrm{h}}=1 / \gamma_{\mathrm{h}}$, from the fits to experimental data using Eq. (7). Their values, depicted in Fig. 2(c), remain nearly constant throughout the investigated detuning range, indicating that the photocarrier and hole spin dynamics are not strongly influenced by the initial energy relaxation of nonresonantly excited carriers. It is also clear that the electron (trion) spin lifetime remains close to the photocarrier recombination time. Using TRPL, we measure a photocarrier recombination time of about $175 \mathrm{ps}$ at a temperature of $15 \mathrm{~K}$, using nonresonant excitation with larger detuning than during the TRKR measurements. As the recombination time increases with detuning, ${ }^{25}$ this value only provides an upper bound for the photocarrier lifetime under the excitation conditions in the TRKR measurements. We may therefore conclude that the electron spin coherence time is mostly limited by the carrier lifetime for weak, nonresonant excitation.

Next, we discuss power-dependent TRKR measurements. For this series, the pump power was increased, relative to the values used in the previous series, by more than two orders of magnitude. Figure 3(a) shows three TRKR traces for different excitation powers. The laser excitation energy was chosen to be near resonant and kept fixed throughout the series; the sample temperature was $10 \mathrm{~K}$. While for weak pumping, the TRKR traces show almost no negative part, it becomes quite pronounced for higher pump powers and the subsequent decay of the negative signal becomes more
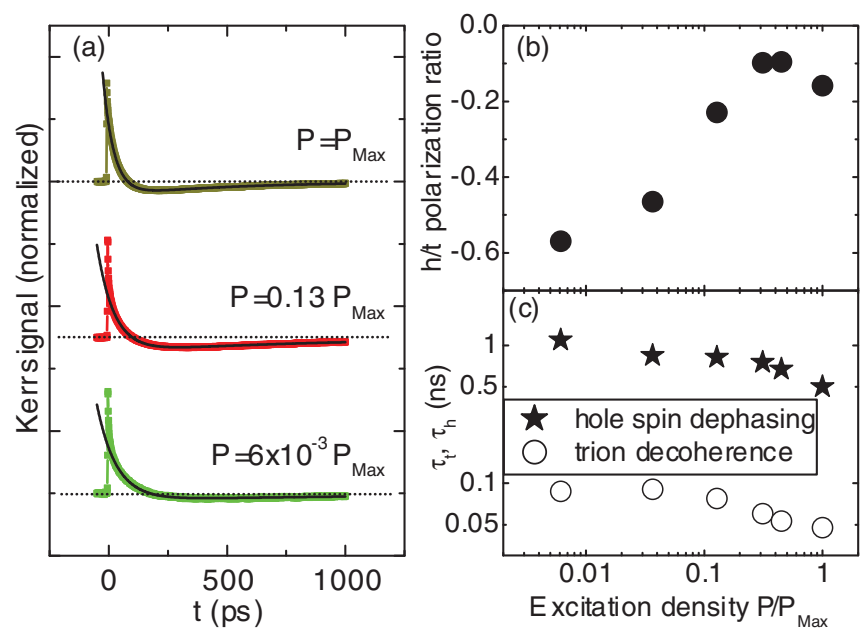

FIG. 3. (Color online) (a) TRKR traces measured at $10 \mathrm{~K}$ with fixed, near-resonant laser excitation energy and various pump powers (symbols). The solid lines represent fits to the data according to the theory (extended to $t<0$ for better visibility). (b) Ratio of hole and trion spin polarizations after rapid initial hole dephasing extracted from the measurements as a function of excitation density. (c) Hole SDT (solid stars) and trion spin decoherence time (open symbols) as a function of excitation density. 
rapid. As in the previous measurement series, our theory closely fits the experimental traces, except for the first few picoseconds, in which the initial hole spin dephasing takes place. From the extracted ratio of hole and electron spin polarization we see that for weak, near-resonant pumping, the hole spin polarization is significantly larger than for stronger pumping [Fig. 3(b)], most likely indicating the importance of spin nonconserving carrier-carrier scattering for rapid hole spin decoherence. We also observe that the long-time hole and trion SDTs decrease as the pump power is increased. This decrease is rather weak (by about a factor of 2 over more than two orders of magnitude of the pulse power) and may be due to sample heating by the pump beam. A rapid decrease of the hole SDT with temperature has been observed previously by several groups. ${ }^{6,11,26}$ The decreasing trion spin lifetime observed in the power-dependent experiments is not limited by faster photocarrier recombination, as we observe in TRPL under nonresonant excitation conditions that the photocarrier recombination time in our sample increases as the temperature is raised, from $150 \mathrm{ps}$ at $4 \mathrm{~K}$ to $400 \mathrm{ps}$ at $40 \mathrm{~K}$. Such an increase is typically observed in the low temperature regime for intrinsic, as well as $p$ - or $n$-doped QW structures. ${ }^{20,27-29}$ Therefore, the reduction of the trion spin lifetime must be caused by spin-related decoherence processes. Most likely, this is due to an increased effective $k$ vector, which leads to larger spin-orbit fields and more rapid dephasing via the Dyakonov-Perel mechanism. ${ }^{30}$

\section{B. Resonant spin amplification}

We now turn to the RSA measurements. All series of measurements were performed at a nominal sample temperature of $1.2 \mathrm{~K}$ in Voigt geometry. In this temperature range, we previously observed hole SDTs above $70 \mathrm{~ns},{ }^{11}$ which exceed the laser repetition period and lead to well-defined RSA signals. Figure 4 shows the two principally different shapes of the RSA traces we observe in experiment: For resonant excitation conditions (bottom curve), in which the optically oriented hole spin polarization is conserved during the photocarrier lifetime, the RSA traces have a characteristic, batwinglike shape, and the maximum at zero magnetic field is absent. This peculiar shape arises from the process in which

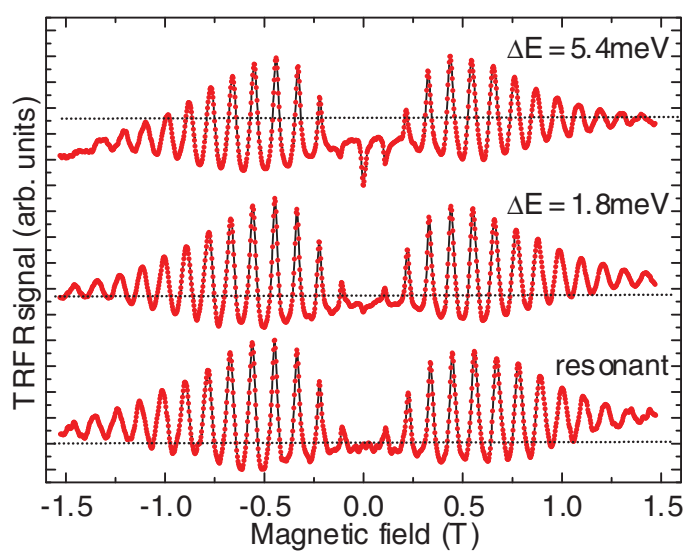

FIG. 4. (Color online) The measured RSA traces for resonant and off-resonant excitation conditions at low excitation power for three different values of pump pulse detuning. the spin polarization of optically oriented holes is turned into the resident hole spin polarization after recombination: ${ }^{10,11}$ At zero magnetic field, the optically oriented hole spin polarization is removed by photocarrier recombination, as described above. With an applied in-plane magnetic field, however, the strongly different $g$ factors of electrons and holes lead to incommensurate precession of the optically oriented spin polarizations, allowing the electrons to recombine with unpolarized, resident holes, so that some hole spin polarization remains in the sample after photocarrier recombination. This hole spin polarization is oriented in the same way as the optically created holes (which we refer to as the positive orientation). If the hole precession frequency is an integer multiple of the laser repetition rate this spin polarization is resonantly magnified to yield pronounced, equally spaced peaks in the RSA signal. Their magnitude grows as the magnetic field increases due to the increased efficiency of the aforementioned initialization process. The decay of the RSA peak height with further increase of the magnetic field stems from hole ensemble dephasing due to the $g$-factor inhomogeneity. ${ }^{25}$

For off-resonant excitation, when a substantial part of the optically oriented hole spin polarization dephases before photocarrier recombination (as discussed in Sec. IV A above), the RSA signal shape becomes more complex, as the middle and top traces in Fig. 4 show. Here we observe RSA peaks also at zero magnetic field, and the amplitude of these peaks initially decreases at low fields. For a certain field, we observe a change of polarity of the RSA peaks, then the RSA peak amplitude first increases, then decreases again. These observations can be understood in the following way: For zero magnetic field, a fast partial re-equilibration of the hole spin polarization leads to negative final polarization of the resident holes due to removal of the optically aligned holes by spin-conserving electrons upon recombination, as discussed in Sec. IV A. As the hole spins are static in the absence of precession at $B=0$, the spin polarization created in this way by subsequent pump pulses constructively interferes, leading to the observed negative zero-field peak. At nonzero fields, this process competes with the spin alignment due to the trion precession discussed above, which effectively leads to randomization of the trion spin orientation at the moment of recombination so that recombination with an oppositely oriented hole becomes possible. As a result of this process, an excess population of optically oriented holes is left behind after recombination, which results in a positive spin orientation. Obviously, this mechanism is only effective at nonzero magnetic fields and its role increases as the field grows. This increasing compensation between these two processes is manifested by reduced negative RSA peak amplitudes at growing fields, visible in the top trace in Fig. 4. At a certain magnetic field, the precession-related hole spin initialization process becomes dominant, leading to a positive spin orientation of the resident holes. Hence, we observe the polarity change in the RSA peaks. For even larger magnetic fields, reduced RSA peak height is again observed due to the inhomogeneous ensemble dephasing.

The competition of the two orientation processes leading to opposite spin polarizations is reflected in our theory by the factor $f$ in Eq. (8a) which (apart from the inhomogeneous dephasing) determines the envelope of the RSA response. The 
hole spin relaxation which tends to re-equilibrate the hole spin polarization after optical creation of extra holes with positive spin orientation (or, equivalently, depletion of the negatively oriented holes by binding them with the optically created excitons into trions) is described by the first two terms in Eq. (8b). Here, according to Eq. (6), $e^{-u}$ is the degree of the fast spin polarization decay during or just after the excitation. The trion precession, which leads to orientation in the positive direction, is accounted for by the third term. It is clear from the form of Eq. (8b) that a sign change of $f$, corresponding to a change of the "polarity" of the RSA response, is possible only for $u>0$, that is, in the presence of initial spin relaxation. Moreover, the position of this transition is shifted to higher fields as $u$ increases, leading to a growing number of inverted RSA peaks in the low field region.

The growing number of inverted peaks is clearly seen in Fig. 4: For increasing laser detuning, first a single negative RSA peak at zero field develops, then additional peaks of the same orientation are seen so that the magnetic field for which a crossover between the initialization mechanisms occurs is increased. This allows us to conclude that the increasing detuning of the pump pulse toward higher energies leads to increased initial spin relaxation. This is, in fact, expected, as off-resonant excitation supplies extra energy to the system leading to additional relaxation processes that usually take place on picosecond time scales and can lead to spin flips.

Our theory allows us to closely model the RSA signal shape using Eq. (8a) integrated over the inhomogeneous distribution of $g$ factors. In the theoretical modeling underlying the fitting, we assume that the intrinsic dephasing rates are constant in the relevant range of the magnetic field. This amounts to assuming that the spectral densities of the reservoir coupled to the hole spins are constant in the corresponding range of frequencies. This is true in particular for an Ohmic reservoir in the high-temperature regime, which is the case here as $\hbar \omega_{\mathrm{h}} \ll k_{\mathrm{B}} T$ in the whole range of magnetic fields studied. Examples of least-squares fits obtained in this way for the positive-field parts of some of the measured RSA traces are presented in Fig. 5(a). The modeling result (blue line) not only reproduces all the features of the experimental RSA traces (red points) but also yields values that are quantitatively close to the measurement results. This good agreement allows us to extract the values of various physical characteristics of the hole spin system. In Fig. 5(b), we show the value of the transverse SDT, $T_{2}=2 / \kappa$. This intrinsic dephasing time increases for decreasing detuning and saturates for low detunings at about $100 \mathrm{~ns}$. The intensities of the fast decoherence and the ratio of hole and trion spin polarization are plotted in Fig. 5(c). The ratio of hole and trion spin polarization reaches -1 as the resonance is approached. This clearly demonstrates that the rapid initial dephasing induced by the off-resonant excitation at low temperatures leads only to a small loss of the optical orientation which disappears completely at resonance. On the contrary, the dephasing factor $w$ remains finite even at the resonance. This is due to the fact that spin dephasing is induced by an optical excitation due to selective coupling of the light field to one of the spin states (according to the selection rules). ${ }^{31}$ From our fitting we conclude that the $g$ factor tends to increase slightly with growing detuning [Fig. 5(d)]. The same holds true for the standard deviation of the ensemble $g$-factor
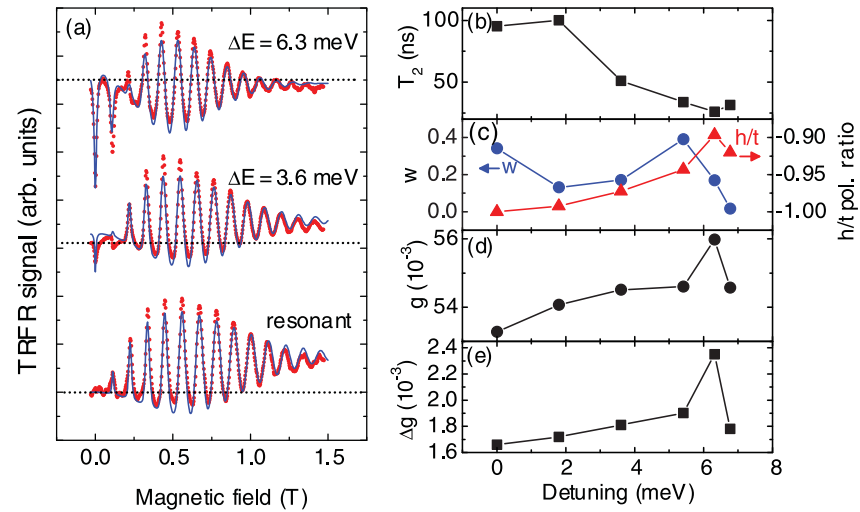

FIG. 5. (Color online) (a) Experimental RSA traces (red points) and best fits according to Eqs. (8a)-(8d) (blue lines) for selected values of the detuning. (b)-(e) Parameter values extracted from the fitting: the dephasing time (b), the fast decoherence parameter and the ratio of hole and electron spin polarization (c), the hole $g$ factor (d), and the standard deviation of the $g$-factor distribution in the ensemble (e). The lines are guides for the eye.

distribution [Fig. 5(e)]. Both of these effects may be explained by a reduced absorption of the pump pulse as the detuning is increased, leading to a reduction of the sample temperature, and a smaller spin-polarized hole ensemble. A shift of the hole $g$ factor to larger absolute values with temperature reduction has already been observed by Syperek et al. ${ }^{10} \mathrm{~A}$ smaller ensemble of spin-polarized holes is more susceptible to $g$ factor inhomogeneity which arises from local fluctuations of, for example, the QW width or the disorder potential.

The theoretical curves turn out to be sensitive to all the model parameters except for $\kappa^{\prime}$. The latter affects the shape of the curves only at very low magnetic fields since, according to Eqs. (8c) and (8d), it can be neglected when $\kappa^{\prime} \ll \omega_{\mathrm{h}}$ which holds already in the vicinity of the first peak. The values of $\kappa^{\prime}$ obtained for nonzero detuning range from 0.012 to $0.03 \mathrm{~ns}^{-1}$, which corresponds to about $50 \%$ of the value of $\kappa=2 / T_{2}$ (except for the largest detuning, where $\kappa^{\prime}$ is lower). This suggests that the spin decoherence is dominated by the in-plane dephasing, described by the rate $\kappa_{z}$, as opposed to the relaxation of the projection on the structure normal (described by $\kappa_{x}$ ). This is expected for heavy holes, as the relaxation of the axial component would involve a spin transfer of $3 \hbar$ and therefore should be suppressed.

As can be seen in Fig. 6(a), similar effects in the RSA traces are observed for increasing pump power at resonant excitation conditions. First, a zero-field peak appears in the RSA traces, then additional peaks are observed for higher pump powers. Again, our theory allows us to precisely model the shape of the experimental RSA traces and to extract the fast dephasing parameters and the hole SDT. Here we attribute the growing fast dephasing, indicated by the reduction of the ratio of hole and trion spin polarization [Fig. 6(c)], to the considerably increased amount of energy pumped into the system. This leads to an increased density of various excitations and, in consequence, to stronger spin nonconserving scattering, in the time window before photocarrier recombination takes place, as observed in the TRKR measurements. An increase of the pump power also 

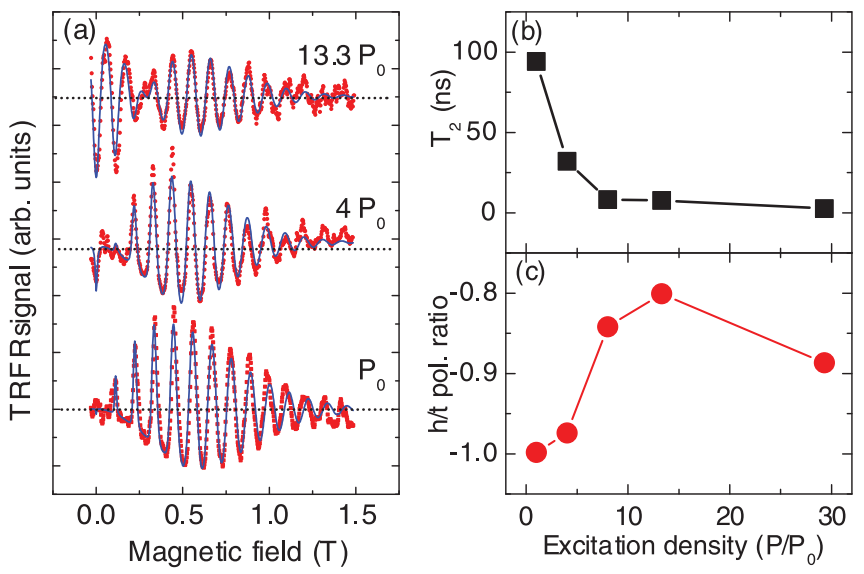

FIG. 6. (Color online) (a) Experimental RSA traces (red points) and best fits according to Eqs. (8a)-(8d) (blue lines) for selected values of the excitation density. Parameter values extracted from the fitting as a function of excitation density. (b) Dephasing time $T_{2}$. (c) Ratio of hole and trion spin polarization.

influences the SDT, $T_{2}$, of the resident hole spins, that is, the spin dynamics after photocarrier recombination, most likely due to sample heating, as can be seen in the drastic reduction of $T_{2}$ [Fig. 6(b)]. This interpretation is supported by the fact that the hole $g$ factor slightly decreases with increasing pump power (not shown), as expected for an increasing sample temperature.

\section{CONCLUSION}

We have performed a time-resolved study of hole spin dynamics in a $p$-modulation-doped QW under different excitation conditions. In TRKR measurements at zero magnetic field, we observed the appearance of a hole spin polarization oriented opposite to the optically oriented holes for nonresonant or high-intensity excitation conditions. In RSA measurements under nonresonant or high-intensity excitation conditions, we observe a competition between two different initialization processes for a resident hole spin polarization, which leads to a complex shape of the RSA traces and to the appearance of inverted spin polarization at low magnetic fields. Negative spin orientation was earlier observed in luminescence from $n$ doped quantum dot systems under off-resonant excitation. ${ }^{32-34}$ Our Kerr rotation and RSA results show that it is possible not only to optically polarize hole spins in a $p$-doped system but also to control the sign of this polarization by changing either the excitation conditions or the magnetic field.

We developed a theoretical model which quantitatively describes the time-resolved Kerr and RSA signals and allows us to attribute the spin polarization at zero field to a decoherence-assisted process in which the hole spin polarization partly relaxes toward equilibrium within a very short time after a high-power or off-resonant excitation. The very good agreement obtained between the measurement data and the model results allows us to extract the parameters relevant to the hole spin dynamics, including the ratio of hole and electron spin polarizations after optical orientation, the intrinsic (homogeneous) spin coherence time $T_{2}$, and the $g$-factor distribution in the ensemble.

Remarkably, rapid initial hole spin dephasing on the few-picosecond time scale and long hole SDTs reaching 100 ns coexist under off-resonant excitation conditions in low magnetic fields at low temperatures. Thus, our findings open the way to optical spin orientation under conditions that assure a long lifetime of the oriented hole spins.

\section{ACKNOWLEDGMENTS}

This work was supported in part by the DFG (Germany) under Grants No. SPP 1285 and No. SFB 689 (M. Kugler, M. Griesbeck, T. Korn, C. Schüller), by the Foundation for Polish Science under the TEAM program, cofinanced by the European Regional Development Fund, (K. Korzekwa and P. Machnikowski), and by a Research Group Linkage Project of the Alexander von Humboldt Foundation (P. Machnikowski and T. Kuhn). The authors would like to thank M. Glazov for highly fruitful discussion. *pawel.machnikowski@pwr.wroc.pl

†tobias.korn@physik.uni-r.de

${ }^{1}$ J. Fabian, A. Matos-Abiague, C. Ertler, P. Stano, and I. Zutic, Acta Phys. Slovaca 57, 565 (2007).

${ }^{2}$ M. W. Wu, J. H. Jiang, and M. Q. Weng, Phys. Rep. 493, 61 (2010).

${ }^{3}$ D. J. Hilton and C. L. Tang, Phys. Rev. Lett. 89, 146601 (2002).

${ }^{4}$ K. Shen and M. W. Wu, Phys. Rev. B 82, 115205 (2010).

${ }^{5}$ T. C. Damen, L. Viña, J. E. Cunningham, J. Shah, and L. J. Sham, Phys. Rev. Lett. 67, 3432 (1991).

${ }^{6}$ P. Schneider, J. Kainz, S. D. Ganichev, S. N. Danilov, U. Rössler, W. Wegscheider, D. Weiss, W. Prettl, V. V. Bel'kov, M. M. Glazov, L. E. Golub, and D. Schuh, J. Appl. Phys. 96, 420 (2004).

${ }^{7}$ C. Lü, J. L. Cheng, and M. W. Wu, Phys. Rev. B 73, 125314 (2006).

${ }^{8}$ B. Baylac, T. Amand, X. Marie, B. Dareys, M. Brousseau, G. Bacquet, and V. Thierry-Mieg, Solid State Commun. 93, 57 (1995).
${ }^{9}$ X. Marie, T. Amand, P. Le Jeune, M. Paillard, P. Renucci, L. E. Golub, V. D. Dymnikov, and E. L. Ivchenko, Phys. Rev. B 60, 5811 (1999).

${ }^{10}$ M. Syperek, D. R. Yakovlev, A. Greilich, J. Misiewicz, M. Bayer, D. Reuter, and A. D. Wieck, Phys. Rev. Lett. 99, 187401 (2007).

${ }^{11}$ T. Korn, M. Kugler, M. Griesbeck, R. Schulz, A. Wagner, M. Hirmer, C. Gerl, D. Schuh, W. Wegscheider, and C. Schüller, New J. Phys. 12, 043003 (2010).

${ }^{12}$ T. Korn, Phys. Rep. 494, 415 (2010).

${ }^{13}$ M. Studer, M. Hirmer, D. Schuh, W. Wegscheider, K. Ensslin, and G. Salis, Phys. Rev. B 84, 085328 (2011).

${ }^{14}$ D. Heiss, S. Schaeck, H. Huebl, M. Bichler, G. Abstreiter, J. J. Finley, D. V. Bulaev, and D. Loss, Phys. Rev. B 76, 241306 (2007).

${ }^{15}$ J. R. Petta, A. C. Johnson, J. M. Taylor, E. A. Laird, A. Yacoby, M. D. Lukin, C. M. Marcus, M. P. Hanson, and A. C. Gossard, Science 309, 2180 (2005). 
${ }^{16}$ J. Fischer, W. A. Coish, D. V. Bulaev, and D. Loss, Phys. Rev. B 78, 155329 (2008).

${ }^{17}$ R. Winkler, S. J. Papadakis, E. P. De Poortere, and M. Shayegan, Phys. Rev. Lett. 85, 4574 (2000).

${ }^{18}$ P. Machnikowski and T. Kuhn, Phys. Rev. B 81, 115306 (2010).

${ }^{19}$ T. Andlauer and P. Vogl, Phys. Rev. B 79, 045307 (2009).

${ }^{20}$ M. Kugler, T. Andlauer, T. Korn, A. Wagner, S. Fehringer, R. Schulz, M. Kubová, C. Gerl, D. Schuh, W. Wegscheider, P. Vogl, and C. Schüller, Phys. Rev. B 80, 035325 (2009).

${ }^{21}$ J. J. Baumberg, D. D. Awschalom, N. Samarth, H. Luo, and J. K. Furdyna, Phys. Rev. Lett. 72, 717 (1994).

${ }^{22}$ J. M. Kikkawa and D. D. Awschalom, Phys. Rev. Lett. 80, 4313 (1998).

${ }^{23}$ I. A. Yugova, M. M. Glazov, E. L. Ivchenko, and A. L. Efros, Phys. Rev. B 80, 104436 (2009).

${ }^{24}$ H.-P. Breuer and F. Petruccione, The Theory of Open Quantum Systems (Oxford University Press, Oxford, 2002).

${ }^{25}$ D. R. Yakovlev and M. Bayer, in Spin Physics in Semiconductors, edited by M. I. Dyakonov (Springer, Berlin, 2008), p. 135.

${ }^{26}$ I. A. Yugova, A. A. Sokolova, D. R. Yakovlev, A. Greilich, D. Reuter, A. D. Wieck, and M. Bayer, Phys. Rev. Lett. 102, 167402 (2009).
${ }^{27}$ J. Feldmann, G. Peter, E. O. Göbel, P. Dawson, K. Moore, C. Foxon, and R. J. Elliott, Phys. Rev. Lett. 59, 2337 (1987).

${ }^{28}$ V. Ciulin, P. Kossacki, S. Haacke, J.-D. Ganière, B. Deveaud, A. Esser, M. Kutrowski, and T. Wojtowicz, Phys. Rev. B 62, R16310 (2000).

${ }^{29}$ P. Olbrich, J. Allerdings, V. V. Bel'kov, S. A. Tarasenko, D. Schuh, W. Wegscheider, T. Korn, C. Schüller, D. Weiss, and S. D. Ganichev, Phys. Rev. B 79, 245329 (2009).

${ }^{30}$ M. I. Dyakonov and V. I. Perel, Zh. Eksp. Teor. Fiz. 60, 1954 (1971) [Sov. Phys. JETP 33, 1053 (1971)].

${ }^{31}$ A. Grodecka, P. Machnikowski, and J. Förstner, Phys. Rev. A 79, 042331 (2009).

${ }^{32}$ A. S. Bracker, E. A. Stinaff, D. Gammon, M. E. Ware, J. G. Tischler, A. Shabaev, A. L. Efros, D. Park, D. Gershoni, V. L. Korenev, and I. A. Merkulov, Phys. Rev. Lett. 94, 047402 (2005).

${ }^{33}$ S. Laurent, M. Senes, O. Krebs, V. K. Kalevich, B. Urbaszek, X. Marie, T. Amand, and P. Voisin, Phys. Rev. B 73, 235302 (2006).

${ }^{34}$ A. Shabaev, E. A. Stinaff, A. S. Bracker, D. Gammon, A. L. Efros, V. L. Korenev, and I. Merkulov, Phys. Rev. B 79, 035322 (2009). 